\title{
Diseño de un Control Regulador para un Sistema de Servo-Posicionamiento Neumático
}

\author{
Iván Ramírez ${ }^{1}$, Christopher Toro ${ }^{1}$, Jonathan Diaz ${ }^{2}$ \\ idramirez@uniquindio.edu.co,christopo217@hotmail.com,jdiazm@inaoep.mx \\ ${ }^{1}$ Universidad del Quindío, Ingeniería Electrónica, Departamento de Automatización y Control, 630004, \\ Armenia, Quindío, Colombia. \\ ${ }^{2}$ Instituto Nacional de Astrofísica, Óptica y Electrónica INAOE, Maestría en Electrónica, 72840, \\ Tonantzintla, Puebla, México.
}

DOI: $10.17013 /$ risti.29.72-88

Resumen: Los actuadores neumáticos son de bajo costo, seguros, limpios y exhiben una alta relación potencia-peso. Esta contribución presenta una estrategia de control novedosa para resolver el problema de regulación de un sistema de servo posicionamiento neumático, que compensa por las no linealidades del sistema, considerando la posición, velocidad y la diferencia de presiones en las cámaras del cilindro neumático como estados de retroalimentación, en lugar de posición, velocidad y aceleración (PVA) como en los controladores más comunes que se encuentran en la literatura. Los experimentos son realizados con un cilindro neumático sin vástago, de $25 \mathrm{~mm}$ de diámetro y una válvula de control proporcional de $5 / 3$ vías. Para probar la eficiencia y aplicabilidad del esquema de control propuesto tanto simulaciones como resultados experimentales se muestran y se discuten. Los resultados demuestran que un alto desempeño en posicionamiento y respuestas simétricas en el movimiento del deslizador en ambas direcciones puede ser logrado.

Palabras-clave: Lazo interno; estados de retroalimentación; sistema no lineal; lazo externo; control regulador.

\section{Regulation Control Design of a Pneumatic Servo-Positioning System}

Abstract: Pneumatic actuators are low-cost, safe, clean, and exhibit a high
power-to-weight ratio. This contribution presents a novel control strategy to solve
the regulation problem of pneumatic servo positioning systems that cope with the
nonlinearities of the system: airflow-pressure relationship through valve orifice
and friction effects between contact surfaces in actuator seals, by considering
the position, velocity and pressure difference in the chambers of the pneumatic
cylinder, as feedback states instead of position, velocity and acceleration (PVA) as
in the most common controllers found in literature. Experiments are conducted
with a 25-mm bore rod-less pneumatic cylinder and a $5 / 3$-way proportional
control valve. To highlight the efficiency and applicability of the proposed control
schemes, simulation, as well as experimental results are provided and discussed. 
The experimental and simulated results from the regulation control strategy proposed, prove that high-positioning performance and symmetrical responses in forward and backward motion are achieved.

Key words: Inner loop; linear feedback states; nonlinear system; outer loop; regulation control.

\section{Introducción}

Los actuadores neumáticos son ampliamente utilizados en el campo de la automatización, la robótica y la manufactura. Debido a sus atributos especiales, los actuadores neumáticos se han convertido en actuadores alternativos en tareas automatizadas de manejo de materiales. La tecnología neumática presenta muchas ventajas, como alta velocidad, alta generación de fuerza, mejor eficiencia, menos mantenimiento y bajos costos operativos. Sin embargo, su precisión relativamente baja les impide competir con sistemas electromecánicos, hidráulicos y magnéticos cuando se necesita una mayor precisión.

De otro lado, los actuadores neumáticos son difíciles de controlar debido a las características no lineales del sistema. Las no linealidades presentes en los actuadores neumáticos son la rigidez muy baja causada por la compresibilidad del aire en las cámaras del cilindro, las variaciones del caudal másico y la baja amortiguación del actuador, lo que dificulta el control preciso del movimiento (Saravanakumar, Mohan \& Muthuramalingam, 2017). Otras no linealidades en los servosistemas neumáticos son la zona muerta de la válvula, saturación, ancho de banda limitado, relación no lineal flujo de aire-presión a través de los orificios de la válvula y los efectos de fricción entre las superficies de contacto en los sellos del actuador, especialmente en aquellas zonas con velocidad cercana a cero (Rahmat, M.F. et al., 2011), que son desfavorables en el diseño de estrategias para el control de servosistemas neumáticos en aplicaciones de posicionamiento de alta precisión (Ramírez, 2013).

Las altas no linealidades presentes en los sistemas neumáticos implica que sus modelos matemáticos se componen de ecuaciones diferenciales no lineales. Estas ecuaciones se caracterizan por no tener una solución explícita y por lo tanto requieren de un tratamiento exclusivo para cada solución. La teoría de los sistemas dinámicos no lineales permitió el acceso a nuevas herramientas matemáticas, capaces de permitir el análisis de modelos de sistemas más complejos con un comportamiento altamente no lineal que incluso puede alcanzar un comportamiento caótico.

En las últimas décadas, el modelado y control de actuadores servo neumáticos ha sido un tema interesante que ha atraído a investigadores de todo el mundo. Se deben identificar una gran cantidad de parámetros desconocidos para lograr una respuesta dinámica cercana a los sistemas reales. Las tres consideraciones principales para obtener el modelo de un sistema de actuador neumático son: (i) la dinámica de la carga, incluido el modelo de fricción porque la fuerza de fricción existente en el actuador neumático lo hace difícil de controlar. Varios investigadores modelaron la fuerza de fricción para implementar compensación por fricción para una alta precisión en el desempeño del controlador. (ii) la presión, los volúmenes y la temperatura del aire en el cilindro, y (iii) el caudal másico a través de los orificios de la válvula (Sobczyk, Perondi \& Suzuki, 2012). 
(Valdiero, Ritter, Rios, \& Rafikv, 2011) presentaron el modelo de un sistema de servo posicionamiento como una combinación de ecuaciones teóricas y métodos de identificación del sistema. (Sorli, Gastaldi, Codina, \& Heras, 1999) analizaron las características dinámicas de un sistema de posicionamiento servo neumático. Mientras que (Takosoglu, Dindorf, \& Laski, 2009) presentaron el modelo teórico global del sistema servo neumático mediante el uso de una válvula proporcional. (Ramírez \& Ibarguen, 2016) presentaron un modelo obtenido a partir de leyes físicas e identificación para obtener los parámetros del flujo compresible a través de los orificios de la válvula proporcional de control direccional. (Najafi, Fathi, \& Saadat, 2009) modelaron la amortiguación del cilindro neumático al final de las carreras. (Ramírez, 2013) presentó un modelo global del sistema de servo posicionamiento neumático con el modelo de fricción LuGre incluido.

Muchos autores han presentado diferentes enfoques de control y algoritmos para un control preciso de los actuadores neumáticos. Entre estos, (Aziz \& Bone, 2000) presentaron un procedimiento de sintonización automático de los parámetros de servo controladores para sistemas neumáticos; (Rao \& Bone, 2008) presentaron una novedosa ley de control de posición no lineal de Múltiple entrada-una salida (MISO) diseñada mediante el uso del método de backstepping para sistemas neumáticos. Un método efectivo de compensación por fricción fue desarrollado en (Ning \& Bone, 2002), que puede agregarse a cualquier estrategia de control. Cuando esta compensación la combinaron con el clásico enfoque de control posición-velocidad-aceleración/posiciónvelocidad (PVA/PV), alcanzaron experimentalmente una precisión de estado estacionario de $\pm 0.01 \mathrm{~mm}$. (Tsai \& Huang, 2008) propusieron un controlador adaptativo basado en la técnica de aproximación de función (FAT) para estimar las incertidumbres variables en el tiempo en los sistemas de servo-posicionamiento neumático. (Gulati \& Barth, 2009) diseñaron un observador de presión basado en Lyapunov para un sistema servo neumático.

El diseño de sistemas de control complejos es difícil, sin embargo, es relativamente fácil utilizar controladores de lógica difusa, que utilizan una base de conocimiento, como un operador humano. A manera de ejemplo, (Gao \& Feng, 2005) desarrollaron un controlador adaptable Proporcional-Derivativo Fuzzy para el sistema de posicionamiento neumático. (Nagi \& Perumal, 2009) optimizaron el controlador difuso para obtener una respuesta de tiempo mínima. (Kaitwanidvilai \& Olranthichachat, 2011) propusieron una metodología robusta de un controlador difuso de formación de bucles para el control de posicionamiento del sistema servo neumático. (Gyeviki, Tóth, \& Rózsahegvi, 2004) presentaron una estrategia de control por modos deslizantes y su aplicación en un sistema de posicionamiento servo-neumático. (Ramírez, 2018) desarrolló una novedosa estrategia de control de seguimiento para el sistema de servo-posicionamiento neumático.

Por lo anterior, aunque muchos artículos cubren el control de los sistemas de servoposicionamiento neumático, los controladores más comunes encontrados en la literatura usan PVA como los estados de realimentación. Esta contribución presenta una novedosa estrategia para resolver el problema de regulación de los sistemas de servo-posicionamiento neumático. El enfoque es novedoso en el sentido de que tiene en cuenta las no linealidades inherentes a los sistemas de servo posicionamiento neumático 
y considera la posición, la velocidad y la diferencia de presión en las cámaras del cilindro neumático como estados de retroalimentación. La estrategia de control diseñada es implementada en ambos: simulación y en el sistema de servo posicionamiento real.

Este documento está organizado de la siguiente manera: la sección 2 presenta una descripción del Sistema de Servo-Posicionamiento Neumático (SSPN) con sus componentes principales y el sistema neumático de prueba utilizado. La sección 3 presenta el modelo global del SSPN, desarrollado en (Ramírez \& Ibarguen, 2016). En la sección 4 se presenta la estrategia de control propuesta en este artículo para resolver el problema de regulación del SSPN, investiga experimentalmente el efecto de la realimentación de la diferencia de presión medida sobre el flujo no lineal a través de los orificios de la válvula de control direccional proporcional de 5/3 vías utilizada. También se deriva la función de transferencia lineal y un modelo de espacio de estado para el SSPN. Esta sección también implementa el control regulador propuesto sobre el sistema neumático real y sobre el modelo de simulación global. La sección 5 concluye el documento con algunos comentarios y declaraciones finales.

\section{Materiales y Métodos}

\subsection{Set-up experimental}

En la Figura 1 se muestra una representación esquemática de la configuración del sistema de servo posicionamiento neumático utilizado. Consiste de una válvula proporcional de 5 puertos (FESTO, MPYE- 5-1/8 HF-O1OB) y un cilindro neumático sin vástago (FESTO, DGCI-25-1250-KF-YSR). El flujo de aire desde el tanque de aire a las cámaras del cilindro se rige en magnitud y dirección por la posición del spool de la válvula. Un actuador electromagnético (un solenoide) acciona el spool de la válvula. La parte electromecánica de la servo válvula se controla mediante un sistema de realimentación con un ancho de banda de aproximadamente $70 \mathrm{~Hz}$, de modo que, en este rango de frecuencia, la posición del spool es aproximadamente proporcional a la señal de entrada. El cilindro sin vástago se usa para garantizar que el área de la sección transversal en ambas cámaras es la misma. El cilindro tiene un sistema de medición de posición integrado y es un sensor de posición de salida digital magnetoestrictivo (FESTO, CANBUS) con una resolución de $50 \mu \mathrm{m}$.

El deslizador interno (el pistón) se mueve hacia adelante y hacia atrás en el cilindro por medio de aire seco aplicado a cada lado mediante el uso de la válvula de control direccional proporcional de 5/3 vías. La transferencia de movimiento al deslizamiento exterior se logra a través de un acoplamiento fijo. Para medir la presión dentro o en la entrada de las cámaras del cilindro, se adaptaron dos conexiones de aire comprimido y se usaron sensores de presión diferencial para medir la diferencia de presión entre las cámaras del cilindro, que es proporcional a la fuerza impulsora. La velocidad se puede obtener a partir de la integración de la de la aceleración obtenida del acelerómetro.

El sistema de adquisición y control utilizado es un NI DAQ PCI 6014 (National Instruments). Es un dispositivo electrónico especialmente diseñado para el desarrollo de control digital y adquisición de datos. Está compuesto por 16 entradas analógicas (ADC) y dos salidas analógicas (DAC). Se utiliza una computadora para proporcionar el 
voltaje de entrada de conducción a la válvula por medio de un convertidor D/A y leer los transductores por medio de convertidores A/D.

\subsection{Diseño del control de regulación}

El objetivo aquí es resolver el problema de regulación del SSPN considerado. Se propone una estrategia de control novedosa que compensa por las no linealidades del flujo de aire a través de los orificios de la válvula de control direccional proporcional de 5/3 vías y la fricción no lineal. La estrategia de control consta de dos partes:

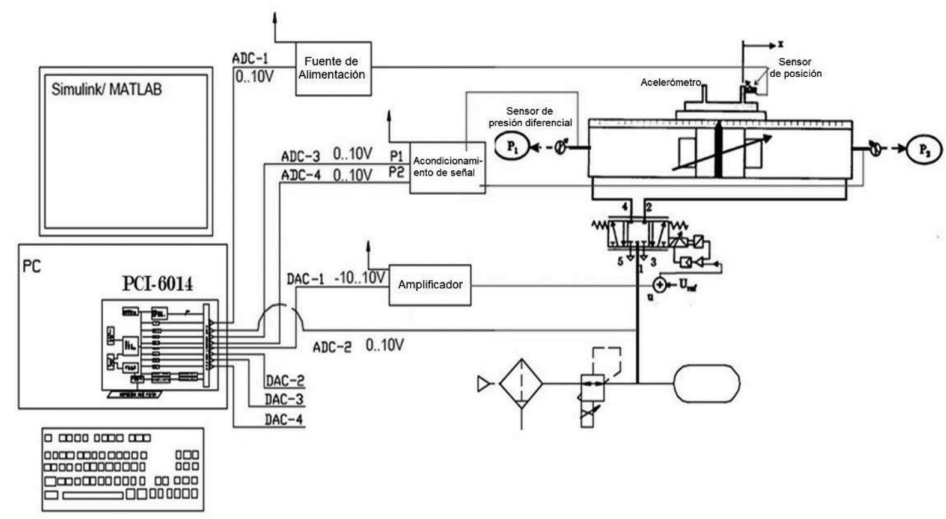

Figura 1 - Set-up experimental del sistema de servo-posicionamiento neumático

Un controlador proporcional (lazo interno) para la diferencia de presión medida en las cámaras del cilindro neumático $\left(P_{1}-P_{2}\right)$, con ganancia proporcional $K_{p}$. Este bucle embebido compensará por las no linealidades de la válvula proporcional, es decir, las no linealidades del flujo a través de los cuatro orificios de la válvula.

Retroalimentación de estados (bucle externo) en el que la posición, la velocidad y la diferencia de presiones medida en las cámaras del cilindro neumático son los estados de retroalimentación. Este lazo compensará por la fricción no lineal para lograr un alto desempeño en posicionamiento.

Para investigar experimentalmente el efecto de la retroalimentación de la diferencia de presiones medida sobre las no linealidades del flujo de aire a través de los orificios de la válvula proporcional, se debe realizar lo siguiente:

Separar el efecto del flujo no lineal desde la fricción no lineal. Esto se logra manteniendo el deslizador en una posición fija, que podría ser a la mitad de la carrera del cilindro neumático, es decir, considerando un subsistema que consiste en la válvula proporcional y dos cámaras aproximadamente iguales en tamaño.

Aplicar un control proporcional para la diferencia de presiones con la compensación por zona muerta de la válvula proporcional dc, como se muestra en la Figura 2. La diferencia de presión deseada $\Delta P_{d}$, es la entrada y la diferencia de presión medida $\Delta P_{m}$, es la salida. 
Identificar la función de transferencia del sistema en lazo o cerrado resultante $G_{c l}(s)$, dada por la ecuación 1:

$$
\mathrm{G}_{\mathrm{cl}}(\mathrm{s})=\frac{\Delta \mathrm{P}_{\mathrm{m}}(\mathrm{s})}{\Delta \mathrm{P}_{\mathrm{d}}(\mathrm{s})}
$$

Teniendo en cuenta los límites de saturación ( \pm 5 voltios) de la ganancia proporcional de la válvula $K_{p}$, se calcula usando uno de los métodos de representación de sistemas usando primer orden más tiempo muerto (POMTM) encontrados en la literatura.

Ahora, para identificar la función de transferencia de lazo cerrado $G_{c l}(s)$ para el subsistema en investigación, se puede estimar la diferencia de presión requerida $\Delta P_{d}$ para la posición deseada, usando el sistema inverso del sistema y suponiendo fricción viscosa. La posición deseada $x_{d}$ se convierte en la entrada al sistema inverso y $\Delta P_{d}$ que es proporcional a la fuerza impulsora requerida es la salida del sistema, como se mostrará en las siguientes secciones. Finalmente, la función de transferencia de lazo cerrado del subsistema $G_{c l}(s)$ se calcula aplicando un modelo de primer orden más tiempo muerto (POMTM).

\section{Resultados}

\subsection{Modelo de un sistema de servo-posicionamiento neumático}

Un actuador neumático se puede modelar a partir de análisis matemáticos teóricos o a partir de la identificación del sistema. La mayoría de los investigadores han utilizado análisis matemáticos teóricos para modelar el actuador neumático, que requiere una combinación de termodinámica, dinámica de fluidos y dinámica de movimiento. Para construir el modelo matemático del SSPN, se deben tener en cuenta tres consideraciones principales: i) las tasas de flujo másico a través de los orificios de la válvula; ii) la presión, el volumen y la temperatura del aire en el cilindro; iii) la dinámica de la carga. El modelado preciso de un actuador neumático es una condición importante tanto para el diseño del control como para optimizar su funcionamiento (Saravanakumar et al., 2017).

Esta sección presenta un modelo de simulación global del SSPN considerado, desarrollado en (Ramírez \& Ibarguen, 2016). El modelo global del sistema de servo posicionamiento neumático consta de tres bloques principales: (i) la servo válvula; (ii) el actuador o cilindro neumático sin vástago y (iii) el deslizador del actuador neumático.

Para describir la dinámica del servo sistema en forma de modelo de espacio de estado, el modelo debe reformularse mediante un conjunto de ecuaciones diferenciales no lineales que describan relaciones únicas entre la entrada, la salida y los estados del sistema.

La representación más apropiada es la ecuación de estado para un sistema dinámico autónomo definido por la ecuación 2.

$$
\dot{x}=f(x, u), x\left(t_{o}\right)=x_{o}
$$


Donde $\mathbf{x} \in \mathbb{R}^{\mathrm{n}}$ es el vector de estados en el tiempo, $\mathrm{t}, \mathbf{u} \in \mathbb{R}^{\mathrm{n}}$ es el vector de entradas, $\mathrm{y}$ $\mathbf{f}: \mathbb{R}^{\mathrm{n}} \rightarrow \mathbb{R}^{\mathrm{n}}$ es el campo vectorial. Como el campo vectorial no depende explícitamente del tiempo, el tiempo inicial siempre se toma como $t_{0}=0$. Por lo tanto, con $x_{1}=P_{1}, x_{2}=P_{2}$, $\mathrm{x}_{3}=\mathrm{y}, \mathrm{x}_{4}=\mathrm{v}, \mathrm{x}_{5}=\mathrm{z}$, el modelo matemático del sistema está definido por las ecuaciones 3 a 5 :

$$
\begin{gathered}
\dot{x}_{1}=\frac{R T}{A x_{3}+V_{1 D}} \dot{m}_{1}-\frac{A x_{1}}{A x_{3}+V_{1 D}} x_{4} \\
\dot{x_{2}}=\frac{R T}{A\left(L-x_{3}\right)+V_{2 D}} \dot{m}_{2}-\frac{A x_{2}}{A\left(L-x_{3}\right)+V_{2 D}} x_{4} \\
\dot{x}_{3}=x_{4}=\frac{A}{M}\left(x_{1}-x_{2}\right)-\frac{1}{M} F_{f} \\
\dot{x}_{5}=x_{4}-\frac{\sigma_{0}\left|x_{4}\right| x_{5}}{g\left(x_{4}\right)} \\
F_{f}=\sigma_{0} x_{5}+\sigma_{1}\left(x_{4}-\frac{\sigma_{0}\left|x_{4}\right| x_{5}}{g\left(x_{4}\right)}\right)+B x_{4} \\
\dot{m}=( \pm) A_{e f f} P_{1} \sqrt{\frac{2 k}{R T(k+1)}}\left[\frac{2}{k+1}\right] \psi\left(\frac{P_{2}}{P_{1}}\right)
\end{gathered}
$$

Donde:

$R$ : es la constante universal del gas.

$T$ :es la temperatura.

$M$ : es la masa deslizante total.

$F$ : es la fuerza total aplicada.

$A$ : es el área efectiva del pistón.

$P_{1}$ : es la presión en la cámara 1.

$P_{2}$ : es la presión en la cámara 2.

$\boldsymbol{V}_{1 D}$ : es el volumen muerto de la cámara 1 , constante.

$\mathrm{A}_{\text {eff: }}$ es el área de sección transversal de los orificios de la válvula.
$\boldsymbol{V}_{2 D}$ : es el volumen muerto de la cámara 2, constante.

$\boldsymbol{\Psi}$ : es el factor de flujo (razón de presiones).

mi : es el flujo másico que entra y/o sale de la cámara 1.

$\mathbf{m}_{\mathbf{2}}$ : es el flujo másico que entra y/o sale de la cámara 2.

$\boldsymbol{\sigma}_{0}$ : es el coeficiente de rigidez de la deformación microscópica de z.

$\boldsymbol{\sigma}_{1}$ : es el coeficiente de amortiguación asociado con $\mathbf{d z} / \mathbf{d t}$.

$\boldsymbol{\sigma}_{2}$ : es el coeficiente de fricción viscoso $(=\mathbf{C})$

$\boldsymbol{y}$ : es la posición del pistón.

z: es la desviación promedio de las rugosidades de la superficie de contacto. 
Para mayores detalles del modelo y de los valores de todas las constantes el lector puede remitirse a (Ramírez \& Ibarguen, 2016).

\subsection{Control de posicionamiento: Regulación}

\section{Identificación de la función de transferencia en lazo cerrado}

La tarea aquí es identificar la función de transferencia de lazo cerrado $G_{c l}(s)$ para el subsistema bajo investigación, que se muestra en la Figura 2. Para esto, la diferencia de presión requerida $\Delta P_{d}$ puede estimarse para la trayectoria de posición invirtiendo el sistema pistón-deslizador y asumiendo fricción viscosa. La posición deseada $x_{d}$ se convierte en la entrada al sistema inverso y $\Delta P_{d}$, que es proporcional a la fuerza de accionamiento requerida es la salida del sistema, como se muestra en la ecuación 6:

$$
\Delta \mathrm{P}_{\mathrm{d}}=\frac{\mathrm{M}}{\mathrm{A}} \ddot{\mathrm{X}}_{\mathrm{d}}+\frac{\mathrm{C}}{\mathrm{A}} \dot{\mathrm{X}}_{\mathrm{d}}
$$

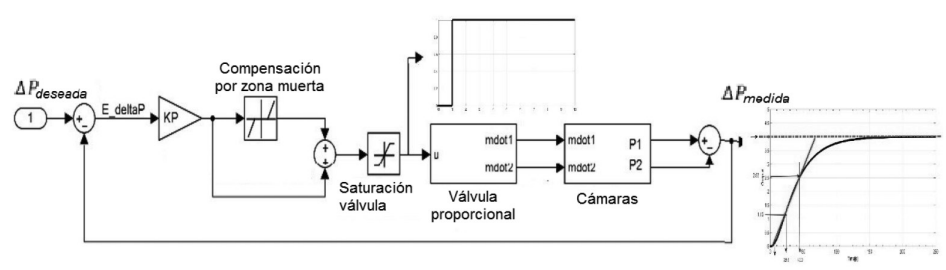

Figura 2 - Diagrama de bloques del subsistema de investigación con un controlador P

Donde M: es la masa deslizante equivalente total, compuesta por la masa del pistón, la masa del deslizador y la masa de un objeto (pieza de trabajo) rígidamente acoplada al deslizador; A: es el área de la sección transversal del pistón, y C: es el coeficiente de fricción viscoso. Finalmente, en la ecuación 7 se presenta la función de transferencia de lazo cerrado del subsistema se identifica aplicando el modelo de primer orden más tiempo muerto (FOPDT) como en (Padma, Srinivas, \& Chidambaram, 2004).

$$
\mathrm{G}_{\mathrm{cl}}(\mathrm{s})=\frac{\Delta \mathrm{P}_{\mathrm{m}}}{\Delta \mathrm{P}_{\mathrm{d}}}=\frac{\mathrm{k}}{\tau \mathrm{s}+1}
$$

Donde $\tau$ es la constante de tiempo $(\tau=0.17 \mathrm{sec})$ y $\mathrm{k}$ es la ganancia fija $(\mathrm{k}=0.67)$. El valor de $K_{p}$ se obtuvo utilizando el método propuesto en (Padma et al., 2004), para diseñar controladores PI/PID en sistemas de primer orden más tiempo muerto (FOPTD) estables o inestables.

\section{Modelo en espacio de estados del sistema de servo-posicionamiento neumático}

El modelo de entrada-salida de un sistema especifica una relación que expresa el cambio de salida como función de entrada, sin considerar la estructura interna y los parámetros del sistema. Para un sistema lineal, de parámetros concentrados, invariante en el tiempo, de única entrada y única salida, la relación entrada-salida consiste en ecuaciones diferenciales lineales de n-ésimo orden con coeficientes constantes, que relacionan la variable de entrada $u(t)$ y la variable de salida $y(t)$. 
Un modelo de entrada-salida implica una función de transferencia operativa que sirve como operador del sistema. La relación de causa y efecto de los sistemas dinámicos lineales invariantes en el tiempo se expresa comúnmente por el concepto de función de transferencia.

Al tener la función de transferencia lineal dada en la ecuación 7, para el subsistema descrito por la ecuación 6 , se puede obtener una función de transferencia lineal completa para el SSPN considerando la fricción viscosa como la única componente existente debida al contacto del pistón con las paredes del cilindro. Esta función de transferencia lineal $\mathrm{G}_{\mathrm{p}}(\mathrm{s})$ está dada en la ecuación 8 y su representación en diagrama de bloques se representa en la Figura 3.

$$
\mathrm{G}_{\mathrm{p}}(\mathrm{s})=\frac{\mathrm{Y}_{\mathrm{m}}(\mathrm{s})}{\Delta \mathrm{P}_{\mathrm{d}}(\mathrm{s})}=\frac{(\mathrm{kA} / \tau \mathrm{M})}{\mathrm{s}^{3}+((\tau \mathrm{C}+\mathrm{M}) / \tau \mathrm{M}) \mathrm{s}^{2}+(\mathrm{C} / \tau \mathrm{M}) \mathrm{s}}
$$

Donde $\Delta \mathrm{P}_{\mathrm{d}}$ es la diferencia de presión deseada, $\mathrm{Y}_{\mathrm{m}}$ es la posición medida, $\mathrm{k}$ y $\tau$ son la ganancia fija y la constante de tiempo del subsistema, respectivamente.

Para describir este sistema dinámico en la forma del modelo de espacio de estado, el modelo debe reformularse mediante un conjunto de ecuaciones diferenciales de primer orden o ecuaciones en diferencia que describan relaciones únicas entre la entrada, la salida y los estados del sistema.

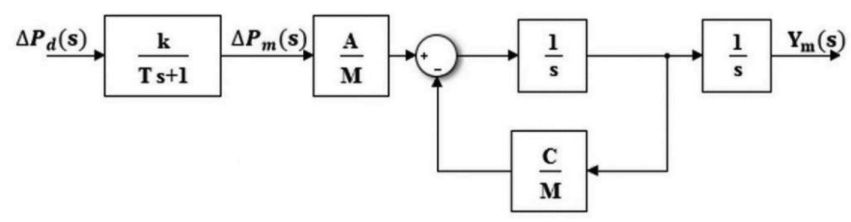

Figura 3 - Modelo lineal entrada-salida del sistema de servo-posicionamiento neumático

El modelo lineal de entrada-salida del SSPN es único y se puede utilizar para caracterizar el sistema a través de un conjunto único de ecuaciones diferenciales de primer orden, como se muestra en las ecuaciones 9:

$$
\begin{gathered}
\dot{\mathrm{x}_{1}}=\mathrm{x}_{2} \\
\dot{x_{2}}=-\frac{C}{M} x_{2}+\frac{A}{M} x_{3} \\
\dot{x_{3}}=-\frac{1}{\tau} x_{2}+\frac{k}{\tau} \Delta P_{d} \\
y_{m}(t)=x_{1} \\
\mathrm{u}(\mathrm{t})=\Delta \mathrm{P}_{\mathrm{d}}
\end{gathered}
$$

A partir de las ecuaciones diferenciales anteriores, el sistema se puede describir en forma de espacio de estado, ecuaciones 10 y 11: 


$$
\left[\begin{array}{l}
\dot{x}_{1} \\
\dot{x}_{2} \\
\dot{x}_{3}
\end{array}\right]=\left[\begin{array}{ccc}
0 & 1 & 0 \\
0 & -\frac{C}{M} & \frac{A}{M} \\
0 & 0 & -\frac{1}{\tau}
\end{array}\right]\left[\begin{array}{l}
x_{1} \\
x_{2} \\
x_{3}
\end{array}\right]+\left[\begin{array}{c}
\mathrm{o} \\
\mathrm{o} \\
\frac{k}{\tau}
\end{array}\right] \Delta P_{d}
$$

Y

$$
y_{m}=\left[\begin{array}{lll}
1 & 0 & 0
\end{array}\right]\left[\begin{array}{l}
x_{1} \\
x_{2} \\
x_{3}
\end{array}\right]
$$

Donde $x_{1}$ es la posición, $x_{2}$ es la velocidad y $x_{3}$ es la diferencia de presiones en las cámaras del cilindro.

\section{Discusión}

En la sección anterior se estableció que un lazo de retroalimentación de la diferencia de presiones medida, embebido con un controlador proporcional y una compensación por zona muerta de la válvula, compensará por las no linealidades del flujo de aire a través de los orificios de la servo válvula.

Posteriormente, se diseña el control del lazo externo, es decir, en los estados en los que la posición, la velocidad y la diferencia de presión medida en las cámaras del cilindro neumático son los estados de retroalimentación. Este lazo compensará por la fricción no lineal para lograr un alto desempeño en posicionamiento.

\subsection{Controlabilidad}

La controlabilidad del modelo de espacio de estado se puede probar a través de la matriz de controlabilidad del sistema $P_{c}$ dada por la ecuación 12. Como el rango de $P_{c}$ es 3 , o equivalentemente det $\left(P_{c}\right)=k^{3} A^{c} / \tau^{3} M^{2} \neq 0$, significa que el sistema es controlable.

$$
P_{c}=\left[B A B A^{2} B\right]=\left[\begin{array}{ccc}
0 & 0 & \frac{k A}{\tau M} \\
0 & \frac{k A}{\tau M} & -\frac{k}{\tau}\left(\frac{C A}{M^{2}}+\frac{A}{\tau M}\right) \\
\frac{k}{\tau} & -\frac{k}{\tau^{3}} & \frac{k}{\tau^{3}}
\end{array}\right]
$$




\subsection{Realimentación de estados}

Con respecto al diseño del lazo externo (retroalimentación del estado) en la estrategia de control propuesta, la ecuación de estado del sistema servo neumático dado en la ecuación 10, puede reescribirse como se muestra en la ecuación 13:

$$
\dot{x}=\boldsymbol{A} x+\boldsymbol{B} u
$$

Con $u=\Delta P_{d}$, se considera la señal de control como una combinación lineal de los estados del SSPN. La retroalimentación lineal de estados está dada por la ecuación 14:

$$
u=-\left[\begin{array}{ccc}
u=-\boldsymbol{K} x \\
\left.\left[\begin{array}{lll}
k_{1} & k_{2} & k_{3}
\end{array}\right]\left[\begin{array}{l}
x_{1} \\
x_{2} \\
x_{3}
\end{array}\right]\right]
\end{array}\right.
$$

Sustituyendo la ecuación 14 en la ecuación de estado del sistema 13, se obtiene la ecuación 15:

$$
\dot{\boldsymbol{x}}=(\boldsymbol{A}-\boldsymbol{B K}) \boldsymbol{x}=\boldsymbol{H} \boldsymbol{x}
$$

Donde $\mathbf{H}$ es la matriz de estado del sistema en lazo cerrado, dada por la ecuación 16:

$$
\boldsymbol{H}=\boldsymbol{A}-\boldsymbol{B} \boldsymbol{K}=\left[\begin{array}{ccc}
s & 1 & 0 \\
0 & -\frac{C}{M} & \frac{A}{M} \\
-\frac{k k_{1}}{\tau} & -\frac{k k_{2}}{\tau} & -\frac{1+k k_{3}}{\tau}
\end{array}\right]
$$

La matriz de estado $\boldsymbol{H}$ del sistema en lazo cerrado desempeña un papel similar a la matriz $\boldsymbol{A}$ para el sistema de lazo abierto, lo que significa que la dinámica del sistema de lazo cerrado está determinada por los valores propios de $\boldsymbol{H}$. El polinomio característico del sistema en lazo cerrado se presenta en la ecuación 18, puede determinarse mediante el determinante de $\boldsymbol{s} \boldsymbol{I}-\boldsymbol{H}$, siendo $\boldsymbol{I}$ la matriz identidad de 3x3, como se muestra en la ecuación 17:

$$
s I-H=\left[\begin{array}{ccc}
s & -1 & 0 \\
0 & s+\frac{C}{M} & -\frac{A}{M} \\
\frac{k k_{1}}{\tau} & \frac{k k_{2}}{\tau} & s+\frac{1+k k_{3}}{\tau}
\end{array}\right]
$$




$$
\operatorname{det}(s I-H)=s^{3}+\left(\frac{\tau C+M\left(1+k k_{3}\right)}{\tau M}\right) s^{2}+\left(\frac{C\left(1+k k_{3}\right)+A k k_{2}}{\tau M}\right) s+\frac{A k k_{1}}{\tau M}
$$

Mediante el diseño adecuado de las constantes de retroalimentación $k_{1}, k_{2}$ and $k_{3}$ se pueden seleccionar valores propios adecuados para la matriz de estado $\boldsymbol{H}$ del sistema en lazo cerrado y, por lo tanto, los polos del sistema en lazo cerrado. La ubicación de polos y el control óptimo son dos métodos básicos para calcular las ganancias de retroalimentación. El método de colocación de polos se usará en este trabajo.

\subsection{Ubicación de polos}

El sistema es controlable, por lo que todas las raíces de la ecuación característica del sistema en lazo cerrado se pueden colocar en cualquier ubicación deseada en el semiplano izquierdo del plano s. La ecuación característica deseada para el sistema de lazo cerrado se puede especificar como en la ecuación 19:

$$
\left(s^{2}+2 \zeta \omega_{n} s+\omega_{n}^{2}\right)\left(s+\zeta \omega_{n}\right)=s^{3}+3 \zeta \omega_{n} s^{2}+\left(2 \zeta^{2}+1\right) \omega_{n}^{2} s+\zeta \omega_{n}^{3}
$$

Debido a que el plano s es infinito, se puede definir una región en el lado izquierdo del plano s complejo donde deben estar las raíces dominantes de la ecuación característica deseada. Por ejemplo, asumiendo que las especificaciones de diseño para una entrada escalón son un tiempo de establecimiento $\boldsymbol{H}_{\boldsymbol{s}}$, (criterio del 2\%) de al menos 2 segundos y un sobre impulso (P.O) inferior al 4\%. Con estas especificaciones pueden escribirse las ecuaciones 20:

$$
\begin{gathered}
T_{s}=\frac{4}{\zeta \omega_{n}}<2.0 \\
\text { P.O. } \approx 100 \exp \left(-\zeta \pi / \sqrt{1-\zeta^{2}}\right)<4.0
\end{gathered}
$$

Resolviendo para $\zeta$ y $\boldsymbol{\omega}_{\mathbf{n}}$ resulta $\zeta>0.72$ y $\boldsymbol{\omega}_{\mathbf{n}}>2.8$, que definen una región en el lado izquierdo del plano s complejo donde deben estar las raíces dominantes de la ecuación de lazo cerrado deseada para cumplir con las especificaciones de diseño establecidas.

Al igualar la ecuación característica del sistema de circuito cerrado (ecuación 18), con la ecuación característica deseada (ecuación 19), se obtienen las siguientes expresiones para las ganancias de retroalimentación $\mathbf{k}_{\mathbf{1}}, \mathbf{k}_{\mathbf{2}}$ and $\mathbf{k}_{\mathbf{3}}$, como se muestra en la ecuación 21.

$$
\begin{gathered}
k_{1}=\frac{\tau M}{A k} \zeta \omega_{n}^{3} \\
k_{2}=\frac{\tau M}{A k}\left(2 \zeta^{2}+1\right) \omega_{n}^{2}-\frac{\tau C}{A k}\left(3 \zeta \omega_{n}-\frac{C}{M}\right) \\
k_{3}=\frac{3 \zeta \omega_{n} \tau}{k}-\frac{\tau C}{k M}-\frac{1}{k}
\end{gathered}
$$




\subsection{Implementación de la estrategia de control propuesta para solucionar el problema de regulación}

La estrategia de control propuesta está implementada en el sistema de servo posicionamiento neumático real. La Figura 4 muestra una representación general del sistema servo neumático y el controlador regulador propuesto.

Las respuestas simuladas de la posición del deslizador ante entradas paso obtenidas usando la estrategia de control propuesta se representan en la Figura 5. Los resultados demuestran que un alto desempeño en posición (bajo tiempo de subida, sobre impulso despreciable y error de estado estacionario insignificante) es alcanzable. Es importante notar la simetría de la respuesta del deslizador en avance $(+250 \mathrm{~mm})$ y en retroceso $(-250 \mathrm{~mm})$.

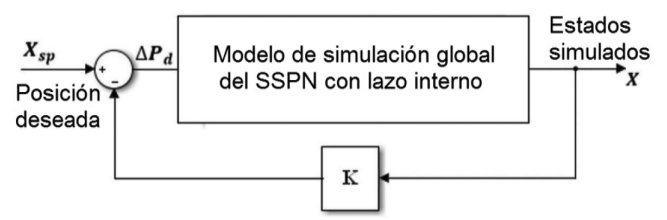

Figura 4 - Representación general para el SSPN y el controlador por regulación propuesto

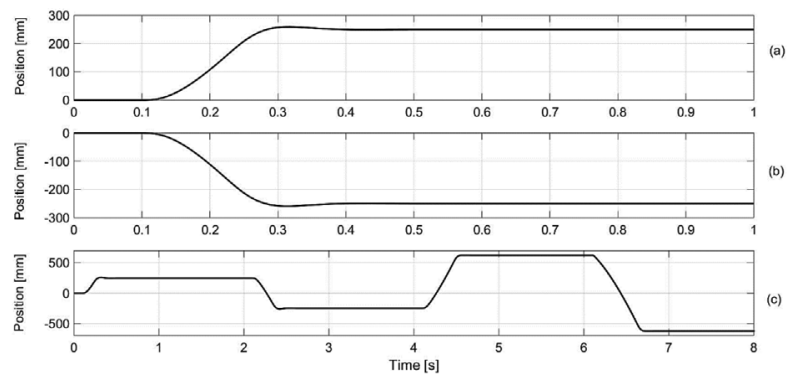

Figura 5 - Respuesta escalón simulada, obtenida mediante la implementación de la estrategia de control de regulación propuesta

La figura 6 muestra una comparación entre la posición obtenida desde el modelo de simulación global y la posición medida en el SSPN experimental, obtenidas para una entrada paso en posición de $500 \mathrm{~mm}$.

\begin{tabular}{lll}
\hline Parámetro de rendimiento & $\begin{array}{l}\text { Respuesta de la posición } \\
\text { simulada }\end{array}$ & $\begin{array}{l}\text { Repuesta de la posición } \\
\text { medida }\end{array}$ \\
\hline $\boldsymbol{t}_{\boldsymbol{r}}(\boldsymbol{s})$ & 0,2105 & 0,2201 \\
\hline $\boldsymbol{t}_{\boldsymbol{s}}(\boldsymbol{s})$ & 0,4037 & 0,3957 \\
\hline $\boldsymbol{O} . \boldsymbol{S}(\%)$ & 0,09 & 0,08 \\
\hline $\boldsymbol{e}_{\boldsymbol{s s}}(\boldsymbol{\mu m})$ & 2,31 & $\sim 50$ \\
\hline
\end{tabular}

Tabla 1 - Parámetros de desempeño simulados y medidos para una entrada escalón de 500 mm en posición 


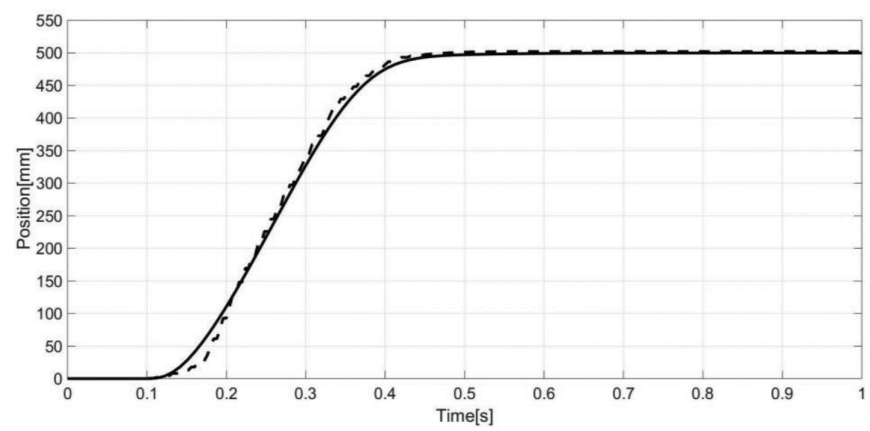

Figura 6 - Comparación entre la posición simulada (línea continua) y la posición medida (línea discontinua) del SSPN obtenido por la implementación de la estrategia de control de regulación propuesta

Los parámetros de desempeño del controlador (100\% respuesta de tiempo de subida $t_{r}$, tiempo de establecimiento $t_{s}$, porcentaje de sobre impulso P.O., y error de estado estacionario $e_{s s}$ ) se muestran en la Tabla 1 para ambos casos simuladas y medidas.

Los resultados demuestran que, con la estrategia de control propuesta en este artículo, el SSPN reproduce con mucha fidelidad el comportamiento del modelo de simulación global. El tiempo de retraso entre los resultados de la simulación y la posición medida es de aproximadamente $0.1 \mathrm{~s}$. Este tiempo de retraso proviene del tiempo muerto introducido por los tubos de conexión entre la válvula y el cilindro y de posibles fugas en las conexiones del cilindro neumático real (la fuga máxima para un nuevo cilindro a 6 bar es de aproximadamente 10 litros/hora). En el modelo de simulación global, los tubos se modelaron como un volumen muerto en el cilindro, a pesar de que las fugas no fueron contempladas en el modelo del SSPN. Si se desea eliminar este tiempo de retraso, una posible alternativa es el uso de dos válvulas proporcionales 3/2. La evaluación de esta alternativa será objeto de futuros trabajos.

Con el fin de analizar la simetría en el comportamiento del deslizador en avance y en retroceso, se aplicaron dos entradas escalón de $250 \mathrm{~mm}$ en direcciones contrarias. La Figura 7 es una comparación entre las posiciones simuladas y medidas que se obtienen implementando la estrategia de control propuesta. Los resultados demuestran que se obtienen respuestas simétricas en el movimiento de avance y retroceso.

\section{Conclusiones}

Una novedosa estrategia de control que considera los dos problemas básicos de control en los SSPN: el flujo de aire no lineal a través de los orificios de la válvula de control direccional proporcional de $5 / 3$ vías y la fricción no lineal en los contactos del pistón con las paredes del cilindro ha sido propuesta y validada. La estrategia de control propuesta consta de dos partes: (i) Controlador proporcional (lazo interno) para la diferencia de presión medida en las cámaras de cilindro $\left(P_{1}-P_{2}\right)$ con ganancia proporcional $K_{p}$, es decir, un lazo de retroalimentación embebida de la diferencia de presiones con compensación DC por zona muerta de la válvula proporcional. Este lazo compensará por las no linealidades de la válvula proporcional, es decir, las no linealidades de la 
ecuación de flujo. (ii) Retroalimentación de estados (lazo externo) en el que la posición, la velocidad y la diferencia de presiones medida en las cámaras del cilindro son los estados de retroalimentación. Este lazo compensará por las no linealidades de la fricción modelada para lograr un alto desempeño del controlador por regulación.
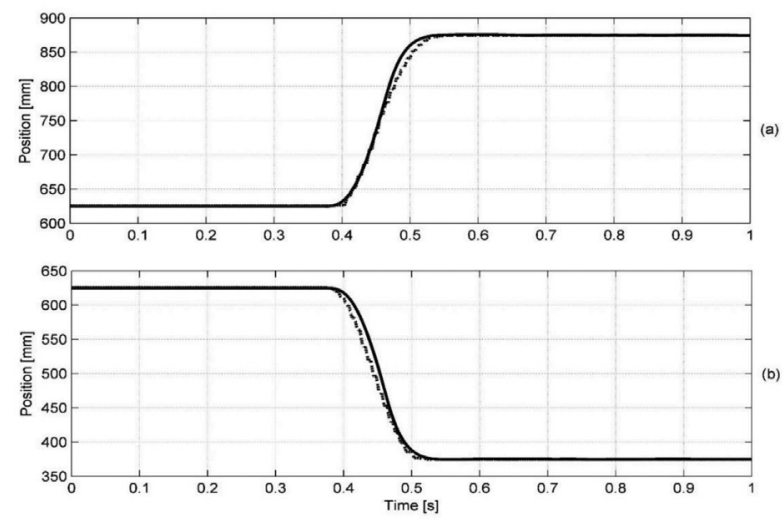

Figura 7 - Comparación entre la posición simulada (línea continua) y la posición medida (línea discontinua) para una entrada de $+250 \mathrm{~mm}$ (a) y $-250 \mathrm{~mm}$ (b)

La principal limitación de la estrategia de control propuesta es el tiempo de retraso que depende de las mangueras de conexión, del caudal de la válvula y del tamaño del cilindro neumático. El tiempo de retraso puede disminuirse utilizando dos válvulas proporcionales de 3/2. La evaluación de esta alternativa será objeto de una próxima publicación. Los resultados experimentales de la estrategia de control de regulación propuesta indican que se puede lograr un alto desempeño en posicionamiento como se muestran en la tabla 1. El bajo sobre impulso se traduce en un alto amortiguamiento en todas las ubicaciones a lo largo del cilindro neumático, un tiempo de subida pequeño y un error en estado estacionario del orden de la resolución del sensor de posición magnetostrictivo usado $(50 \mu \mathrm{m})$.

\section{Referencias}

Aziz, S., \& Bone, G. M. (2000). Automatic tuning of pneumatic servo actuators. Advanced Robotics, 13(6), 563-576.

Gao, X., \& Feng, Z. J. (2005). Design study of an adaptive Fuzzy-PD controller for pneumatic servo system. Control Engineering Practice, 13(1), 55-65.

Gulati, N., \& Barth, E. J. (2009). A globally stable, load-independent pressure observer for the servo control of pneumatic actuators. IEEE/ASME Transactions on Mechatronics, 14(3), 295-306. 
Gyeviki, J., Tóth, I. T., \& Rózsahegvi, K. (2004). Sliding mode control and its application on a servopneumatic positioning system. Transactions on Automatic Control and Computer Science, 49(63), 1-6.

Kaitwanidvilai, S., \& Olranthichachat, P. (2011). Robust loop shaping-fuzzy gain scheduling control of a servo-pneumatic system using particle swarm optimization approach. Mechatronics, 21(1), 11-21.

Nagi, F., \& Perumal, L. (2009). Optimization of fuzzy controller for minimum time response. Mechatronics, 19(3), 325-333.

Najafi, F., Fathi, M., \& Saadat, M. (2009). Dynamic modelling of servo pneumatic actuators with cushioning. The International Journal of Advanced Manufacturing, 42(7), 757-765.

Ning, S., \& Bone, G. M. (2002). High steady-state accuracy pneumatic servo positioning system with PVA/PV control and friction compensation. In IEEE International Conference on Robotics and Automation, 2824-2829.

Padma, R., Srinivas, M. N., \& Chidambaram, M. (2004). A simple method of tuning PID controllers for stable and unstable FOPTD systems. Computers \& Chemical Engineering, 28(11), 2201-2218.

Rahmat, M. F., Sunar, N. H., Najib-Salim, S., Mastura-Shafinaz, Z. A., Mohd-Fauzi, A. A., \& Ismail, Z. H. (2011). Review on modelling and controller design in pneumatic actuator control system. International Journal on Smart and Intelligen Systems, 4(4), 630-661.

Ramírez, I. (2013). Modelling and tracking control of a pneumatic servo positioning system. In II international Congress of Engineering Mechatronics and Automation (CIIMA).

Ramírez, I. (2018). Design of a tracking controller of a siso system of pneumatic servopositioning. Ingeniería y Desarrollo, 36(1), 74-96.

Ramírez, I., \& Ibarguen, F. (2016). Nonlinear control of a servo-pneumatic positioning system. Scientific and technical report No 695. Universidad del Quindío, Colombia.

Rao, Z., \& Bone, G. M. (2008). Nonlinear modeling and control of servo pneumatic actuators. IEEE Transactions on Control Systems Technology, 16(3), 562-569.

Saravanakumar, D., Mohan, B., \& Muthuramalingam, T. (2017). A review on recent research trends in servo pneumatic positioning systems. Precision Engineering, 49, 481-492.

Sobczyk, M. R., Perondi, E. A., \& Suzuki, R. M. (2012). Feedback linearization control with friction compensation applied to a pneumatic positioning system. $A B C M$ Symposium Series in Mechatronics, 5, 252-261.

Sorli, M., Gastaldi, L., Codina, E., \& De las Heras, S. (1999). Dynamic analysis of pneumatic actuators. Simulation Practice and Theory, 7(5), 589-602. 
Takosoglu, J. E., Dindorf, R. F., \& Laski, P. A. (2009). Rapid prototyping of fuzzy controller pneumatic servo-system. The international Journal of Advanced Manufacturing, 40, 349-361.

Tsai, Y. C., \& Huang, A. C. (2008). FAT-based adaptive control for pneumatic servo systems with mismatched uncertainties. Mechanical Systems and Signal Processing, 2(6), 1263-1273.

Valdiero, A. C., Ritter, C. S., Rios, C. F., \& Rafikov, M. (2011). Nonlinear mathematical modelling in pneumatic servo position applications. Mathematical Problems in Engineering, 2011, 1-16. 